\title{
Médiévales
}

Langues, Textes, Histoire

68 | printemps 2015

Langues d'Angleterre

\section{Les livres de Jean Durand († 1416), «physicien » et astronome}

Jean Durand (ob. 1416), Physician and Astronomer, and His Books

\section{Donatella Nebbiai}

\section{(2) OpenEdition}

1 Journals

\section{Édition électronique}

URL : https://journals.openedition.org/medievales/7483

DOI : 10.4000/medievales.7483

ISSN : $1777-5892$

Éditeur

Presses universitaires de Vincennes

\section{Édition imprimée}

Date de publication : 15 juin 2015

Pagination : 93-118

ISBN : 978-2-84292-430-0

ISSN : 0751-2708

\section{Référence électronique}

Donatella Nebbiai, «Les livres de Jean Durand († 1416), «physicien » et astronome », Médiévales [En ligne], 68 I printemps 2015, mis en ligne le 15 juin 2017, consulté le 22 avril 2022. URL : http://

journals.openedition.org/medievales/7483; DOI : https://doi.org/10.4000/medievales.7483 

Donatella Nebbiai

\section{Les livres de Jean Durand ( $† 1416)$, "physicien » et astrologue}

Jean Durand est un clerc normand, sans doute originaire du diocèse de Rouen ${ }^{1}$. Né vers le milieu du XIv ${ }^{e}$ siècle, à une date qu'il nous a été impossible de préciser, mort en $1416^{2}$, il a été chanoine de Notre-Dame de Paris. Astronome et médecin, Durand est actif à Paris à l'époque où Charles V, féru d'astrologie, œuvre pour contrôler l'exercice de cette discipline dans la capitale, oriente la formation des intellectuels qui l'entourent et crée la bibliothèque royale. Les livres, symboles du statut social, sont très appréciés dans ce milieu raffiné, qui cultive la littérature en langue vernaculaire et redécouvre les classiques latins et l'histoire ${ }^{3}$.

1. L'inventaire des livres de Jean Durand, ici étudié, a été présenté lors du séminaire thématique «Materia medica: circulation des livres et construction des savoirs au Moyen Âge et à la Renaissance» (Paris, Institut de recherche et d'histoire des textes, 28 mars 2013). Je remercie très vivement Jean-Patrice Boudet, qui a bien voulu relire cet article, pour ses conseils et ses suggestions.

2. E. WiCKERSHEIMER, Dictionnaire biographique des médecins en France au Moyen Âge, Paris, 1936, réimpr., Genève, 1979, p. 395; D. JACQUART, Supplément au Dictionnaire biographique des médecins..., Genève, 1979, p. 154; EAD., La Médecine médiévale dans le cadre parisien, Paris, 1998, p. 456-457; A. Le Roux DE Lincy, L.-M. Tisserand, Paris et ses historiens aux XIV et XV siècles, Paris 1867, p. 358; J.-P. BoudET, "Charles V, Gervais Chrétien et les manuscrits scientifiques du collège de Maître Gervais», Médiévales, 52 (2007), p. 15-38 (p. 23-24). A. MoLINIER, Obituaires de la province de Sens et de Paris, Paris 1902, t. I, p. 219, indique à tort comme date de mort pour ce personnage l'année 1410 . On ne confondra pas notre Jean Durand avec son homonyme, originaire du diocèse de Tours, qui, à peu près à la même époque, fut docteur en décret et recteur de l'Université de Paris (cf. H. Denifle, E. Châtelain, Chartularium universitatis Parisiensis, Paris, 1889-1897, t. III, p. 84 et 258$)$.

3. Ample bibliographie; voir en priorité A. Vernet éd., Histoire des bibliothèques françaises. I. Les bibliothèques médiévales du ve siècle à 1530, Paris, 1989, p. 267-310; J. Verger, Les Gens de savoir en Europe à la fin du Moyen Âge, Paris, 1997, surtout p. 8599. Cf. aussi D. NeBbiaI, Le Discours des livres. Bibliothèques et manuscrits en Europe, IX ${ }^{\mathrm{e}}$ XV siècles, Rennes, 2013, p. 207-216. 
Cette étude retrace la biographie et les intérêts de Durand. Les sources fragmentaires jusqu'ici connues sur lui peuvent désormais être précisées grâce à la découverte, aux Archives nationales, d'un inventaire après décès de ses biens meubles, daté du 4 au 16 juillet $1416^{4}$. C'est à partir de cet important document, dont on n'édite ici que les sections concernant les livres, les documents administratifs et les objets d'art ${ }^{5}$, que l'on peut tenter de reconstituer ce qui fut son cadre de vie, dans un luxueux hôtel du cloître de Notre-Dame de Paris. Apparaissent aussi les relations que Durand a entretenues aussi bien avec ses confrères qu'avec des membres de la famille royale, et tout particulièrement avec le duc de Bourgogne, Philippe II le Hardi (1404), dont il fut le «physicien».

\section{La carrière}

C'est en 1379 que Charles V, déjà entouré par des astrologues et par des médecins de renom comme Thomas Pizan, le père de Christine, et Gervais Chrétien, doyen de la faculté de médecine en 1359, attribue à Jean Durand une bourse de professeur d'astrologie ${ }^{6}$. À cette époque, le collège, dont Gervais Chrétien, justement, avait entrepris la fondation en 1370, est désormais bien implanté dans la capitale; il vient d'ailleurs d'être doté de statuts officiels ${ }^{7}$. Cette institution était au départ surtout vouée aux études d'arts libéraux et de théologie, et offrait à des étudiants respectivement quatre et six bourses pour ces disciplines. Il revient sans doute au souverain, qui favorise le développement immobilier du collège, d'avoir aussi contribué à y impulser les études d'astrologie; il y fait en tout cas verser, en mai 1372, les livres de philosophie et de médecine provenant

4. Paris, AN, S 851B, $\mathrm{n}^{\circ} 4$ et $\mathrm{n}^{\circ} 4 \mathrm{bis}$; le premier document, $\mathrm{n}^{\circ} 4$, est l'inventaire après décès, qui a été consigné par un seul copiste sur un fascicule de 46 p. écrit sur 34 longues lignes avec réclames, composé de trois cahiers en papier; le $n^{\circ} 4$ bis est une charte d'avril 1418, où son neveu, lui aussi nommé Jean Durand, règle un point résiduel de la succession du défunt avec des personnalités du chapitre de Notre-Dame.

5. $C f$. Annexe, Document $\mathrm{n}^{\circ} 3$.

6. D'après le témoignage de Simon Phares, les bourses étaient au nombre de deux, mais nous ignorons le nom du titulaire de la seconde (J.-P. BoudET, «Charles V, Gervais Chrétien...», p. 22). Cf. H. Denifle, E. Châtelain, Chartularium..., t. II, p. 265 («Rotulus artistarum nationis Normanniae. [...]. Johanni Durandi, cler. Rothomagensis diocesis, mag. in art., scol. in med. secundo anno et legenti Parisius astrologiam ex praecepto domini Regis »). Les liens entre médecine et astrologie sont complexes et échappent à toute généralisation.

7. Statuts en 1378; $c f$. J.-P. Boudet, «Charles V, Gervais Chrétien...», p. 22; P. de Longuemare, «Le collège de maître Gervais Chrétien à Paris », Bulletin de la Société des Antiquaires de Normandie, 31 (1916), p. 182-329; D. JACQUART, La Médecine médiévale..., p. 456-457. 
de la succession de son chirurgien, Jean de Pantalye. Leurs titres nous sont inconnus, mais le document spécifie leur valeur, 40 sous parisis ${ }^{8}$.

Durand, déjà titulaire de la maîtrise ès arts, est alors encore en deuxième année de médecine ${ }^{9}$. Mais Philippe le Hardi l'a déjà remarqué et entend s'assurer de sa collaboration.Aussi, en 1386, le duc de Bourgogne lui accorde-t-il une gratification de 100 francs d'or, tant pour l'aider à obtenir son titre universitaire de médecine que pour «se l'attacher davantage à l'avenir ${ }^{10}$ ». Jean devient alors officiellement «physicien» du duc et le reste jusqu'à la mort de ce dernier, survenue en 1404. C'est avec ce titre qu'il figure, dès 1387, dans le rouleau de la faculté de médecine de l'Université de Paris ${ }^{11}$, et il l'est encore en 1403 lorsqu'il est mentionné parmi les non regentes de la faculté ${ }^{12}$. Sa fonction officielle auprès de Philippe le Hardi lui vaut aussi, en octobre 1392, alors qu'il séjourne au Mans, d'être appelé au chevet de Charles VI, dont la maladie venait de se déclarer, et de recevoir des gages à ce titre ${ }^{13}$. Le document décrit le sceau dont Jean Durand s'était doté: il représente un personnage en buste aux longs cheveux, tenant un soleil inscrit dans un ovale, avec l'inscription Sigillum Johannis Duranti.

Jean Durand a possédé de nombreux bénéfices ecclésiastiques ${ }^{14}$. Sous-diacre de Rouen, il est, en 1387, en passe d'obtenir le titre de chanoine

8. J.-P. Boudet, «Charles V, Gervais Chrétien...», p. 20 (d'après Paris, AN, M 163, $\mathrm{n}^{\circ}$ 11). Gervais Chrétien et sa fondation sont mentionnés dans le Recueil des plus célèbres astrologues de Simon Phares (éd. J.-P. BoudeT, Paris, 1999; $c f$. en particulier les notices dédiées à Gervais Chrétien, qui fut premier médecin de Charles V, et à Charles V lui-même). Pour la bibliothèque du collège de Maître Gervais et les legs de livres en sa faveur (notamment ceux de Guillaume Baron en 1378, de Jean de Neuilly Saint-Front en 1402 et de Guillaume Vauchis, en 1414, aucun legs en sa faveur en revanche n'est attesté de la part de Jean Durand), cf. K. ReBMeISTER-KLeIn, Les Livres des petits collèges à Paris aux XIVe et XVe siècles, thèse de l'Université Paris I sous la direction de J.-P. Genet, 2005, t. II, p. 685-695. Les statuts du collège de Maître Gervais sont transmis dans le ms. Paris, BnF, lat. 4397 A; voir, aux fo 21, $45 \mathrm{v}^{\circ}$ et 82 , les statuts relatifs à la conservation des livres du collège de maître Gervais; L. Delisle, Le Cabinet des manuscrits de la Bibliothèque impériale (puis nationale), Paris, 1868 , t. I, p. 44, n. 15 et 16 (extraits des statuts).

9. Référence citée supra: H. Denifle, E. Châtelain, Chartularium..., t. II, p. 265.

10. Paris, BnF, Collection de Bourgogne 26, $\mathrm{f}^{\circ} 29:$ : [...] l'ayder faire sa faite de maître en médecine et commencer ses cours en cette qualité, reconnaître ses services et se l'attacher davantage à l'avenir. » Cf. P. M. DE WINTER, La Bibliothèque de Philippe le Hardi duc de Bourgogne (1364-1404), Paris, 1985, p. 25.

11. «Rotulus facultatis medicinae», $c f$. H. Denifle, E. Châtelain, Chartularium..., t. III, p. 449: «Item Johanni Durandi, subdyac. Rothomagensis, magister in art. et med. domini ducis Burgundie phisico, de canonicatu ecclesie Rothomagensis. »

12. H. Denifle, E. Châtelain, Chartularium..., t. IV, p. 79. On indique aussi, dans ce document, le nom du médecin du roi et de la reine, qui était alors Richard de Baudribosc.

13. G. Demay, Inventaire des sceaux de la collection Clairambault à la Bibliothèque nationale, Paris, $1885-1886$, t. I, $\mathrm{n}^{\circ} 3251$, p. 344.

14. Pour une étude d'ensemble, $c f$. R. Gane, Le Chapitre de Notre-Dame de Paris au XIV siècle. Étude sociale d'un groupe canonial, Saint-Étienne, 1999, n² 238, d'après Paris, AN, LL 109 A, registres capitulaires de Notre-Dame. Ces données sont désormais à compléter 
de cette cathédrale ${ }^{15}$, à laquelle avaient été également rattachées plusieurs autres personnalités proches de l'entourage royal, comme Gervais Chrétien, déjà cité, et Nicole Oresme ${ }^{16}$. Quelques années plus tard, en 1391, Durand possède aussi le titre de chanoine de Cambrai, dispensé de résidence. Cette prébende lui sera confirmée en 1401, en 1402 et en 1404, sous le gouvernement épiscopal de Pierre d'Ailly (1398-1420) ${ }^{17}$. Toujours à cette époque, grâce au patronage du duc Philippe le Hardi, il obtient la prévôté de Saint-Barthélemy de Béthune ${ }^{18}$, et le duc lui accorde en outre une parcelle d'un bois de chênes situé à Beuvry ${ }^{19}$. Dès 1403 , il possède des prébendes à Coutances et demande à Benoît XIII d'en obtenir aussi à Tournai, à Chartres ou à Saint-Pierre de Lille ${ }^{20}$. En 1404, Durand dispose aussi d'une prébende à Châlons-en-Champagne; il est archidiacre de cette cathédrale en $1406^{21}$.

par celles réunies par M. Maillard-Luypaert et S. Guilbert, respectivement pour les diocèses de Cambrai et de Châlons-en-Champagne, dans le cadre du programme de recherche «Fasti Ecclesiae Gallicanae», initié par H. Millet (http://fasti.univ-paris1.fr/). Signalons en outre que Jean Durand possédait aussi des bénéfices à Créteil, comme en témoignent des extraits de son inventaire après décès ( $c f$. Annexe, Document n $\left.{ }^{\circ} 3\right)$.

15. H. Denifle, E. Châtelain, Chartularium..., t. III, p. 449, «Rotulus facultatis medicine», 1387, cité supra.

16. Cf. Rouen, AD de la Seine-Maritime, G 2115 (8 octobre 1367, réception au chapitre de Gervais Chrétien; 11 février 1370, réception à la prébende canoniale de Nicole Oresme; 28 août 1372, délibération en faveur d'Oresme pour qu'il achève le Liber politicorum qu'il est train de composer pour le roi; 4 mars 1369, restitution par Oresme de quatre volumes qu'il avait empruntés); voir aussi, pour Nicole Oresme, G 2119 (1384, Charles V demande au chapitre de permettre à N. Oresme de continuer à bénéficier de l'hôtel canonial; 1387, prestation de serment comme évêque de Lisieux). $C f$., pour le détail de ces documents, Rouen. Archives départementales de Seine-maritime. Inventaire sommaire, t. II, Paris 1874, passim.

17. Cambrai, BM, ms. 1055, fo 81 et $107 \mathrm{v}^{\circ}$; Città del Vaticano, ASV, Reg. Suppl. 98, $\mathrm{f}^{\circ} 163 \mathrm{v}^{\circ}$, et Reg. Suppl. 96, $\mathrm{f}^{\circ} 54 \mathrm{r}^{\circ}$. Sur Pierre d'Ailly, $c f$. J.-P. Boudet, «Un prélat et son équipe de travail à la fin du Moyen Âge. Remarques sur l'œuvre scientifique de Pierre d'Ailly», dans D. Marcotte éd., Humanisme et culture géographique à l'époque du concile de Constance, Turnhout, 2002, p. 127-150; L. SMoller, History, Prophecy and the Stars : the Christian Prophecy of Pierre d'Ailly, 1350-1420, Princeton, 1994.

18. $C f$. Dijon, AD de la Côte d'Or, B $1852, \mathrm{f}^{\circ} 1 \mathrm{r}^{\circ}, 2 \mathrm{v}^{\circ}, 14 \mathrm{r}^{\circ}$, cité par P. DEWINTER, $L a$ Bibliothèque de Philippe le Hardi..., p. 25.

19. Lille, AD du Nord, B 1213; Mémoires de l'Académie de Dijon, t. 59, $2^{\mathrm{e}}$ série, VII, p. 167-168; Archives historiques et littéraires, II, 1889-1890, p. 342. Pour la protection que Philippe le Hardi accorde à Jean Durand, $c f$. aussi M. MARChAL-VerdDodT, Table des noms de personne et de lieu mentionnés dans les plus anciens comptes de la recette générale de Philippe le Hardi (1383-1389), Bruxelles, 1971, p. 151.

20. Città del Vaticano, ASV, Reg. Suppl. $98, \mathrm{f}^{\circ} 163 \mathrm{v}^{\circ}$ (AVB XXVII, $\mathrm{n}^{\circ} 3065$ ); voir également H. Denifle et E. Châtelain, Chartularium.... t. IV, p. 79 ( «Johanni Durandi, diac. Rothomagens. dioc., in art. et med. mag. dominique ducis Burgundie phisico, de can. ecclesie Tornacensis vel Carnotensis aut S. Petri Insulensis, Tornacensis diocesis»). La possession par Durand de prébendes à Coutances (précisément à Trelly) et non pas à Constance, est confirmée par l'un des documents administratifs décrits dans son inventaire après décès, $c f$. Annexe, Document $\mathrm{n}^{\circ} 3$.

21. Città del Vaticano, ASV, Reg. Suppl. $96, \mathrm{f}^{\circ} 54 \mathrm{r}^{\circ}$, et Reg. Avenion. $322, \mathrm{f}^{\circ} 390 \mathrm{r}^{\circ}$ (AVB $\mathrm{XIX}, \mathrm{n}^{\circ}$ 504). 
Durand obtient encore le titre de chanoine de Châlons. Cette cathédrale possédait, en ces années-là, un remarquable trésor, où Durand emprunte quelques pièces, comme en témoigne un inventaire établi en $1410^{22}$. Rappelons que l'importance qu'avait alors le chapitre de Châlons est confirmée par la présence en son sein de personnalités comme le cardinal Louis de Bar, fils de Robert $\mathrm{I}^{\mathrm{er}}$ et de Marie de France, évêque de Langres et de Poitiers ${ }^{23}$, ou comme l'évêque de Châlons, Charles de Poitiers; ces personnages sont mentionnés comme étant ses débiteurs dans l'inventaire après décès que nous étudions $\mathrm{ici}^{24}$.

Toutefois, le titre ecclésiastique le plus important que Jean Durand a possédé est bien celui de chanoine de Notre-Dame de Paris. Au temps de l'évêché de Pierre d'Orgemont, la cathédrale est le premier centre du pouvoir religieux dans la capitale et le chapitre entretient d'étroits rapports avec la monarchie. Au sein de la cathédrale, la chapelle royale symbolise ces liens; son trésorier, notamment, est aussi le bibliothécaire du chapitre. C'est Jean Durand qui a assuré cette charge jusqu'à sa mort, survenue en juillet 1416. Un registre capitulaire conservé à la bibliothèque de l'Arsenal rapporte, pour les premiers mois de 1417, que son successeur, dont le nom n'est pas indiqué, s'engage à remettre au chapitre la clef de la librairie, qu'il vient d'avoir en concession, pour le cas où il n'aurait plus le titre de chanoine de Paris ${ }^{25}$. Essayons à présent de préciser l'activité de Durand, en particulier au sein du chapitre, et d'évoquer les personnalités qu'il a fréquentées ; toutes, comme on va le voir, sont en rapport avec l'Université (et notamment avec la faculté de médecine) ou avec des membres de la famille royale.

\section{Le milieu}

Jean Gerson, chancelier de l'Université de Paris, lui aussi protégé de Philippe le Hardi, est sans doute la personnalité la plus en vue que Jean Durand ait comptée dans ses relations. Nous savons que Gerson est très apprécié dans le milieu de la faculté de médecine et qu'il a défendu la

22. Extraits reproduits dans 1'Annexe, Document $\mathrm{n}^{\circ} 1$. L'inventaire du trésor de la cathédrale de Châlons est conservé à Reims, AD de la Marne, Chapitre Saint-Étienne de Châlons, G 454 (olim Arm. 1, liasse 53), n 10; le document a été édité par M. PÉLICIER, Inventaire des meubles et joyaux de la cathédrale de Châlons en 1410, Paris 1887, extrait du Bulletin chronologique des travaux historiques et scientifiques, 2 (1886).

23. G. Poull, La Maison souveraine et ducale de Bar, Nancy, 1994.

24. Voir Annexe, Document $n^{\circ} 3$ (éd. des passages à la p. 41 de l'inventaire: «Lettres inventoriées »).

25. «[10 février 1417] Domino thesaurario sacrae cappellae regis qui est nunc canonicus parisiensis, concessa est una clavis librariae ecclesiae parisiensis sic quod, si desierit esse canonicus parisiensis, reddat clavem capitulo. » $C f$. Paris, Arsenal, ms. 6559, I, p. VIII. 
pratique de la médecine en attaquant les charlatans ${ }^{26}$. On connaît aussi les mises en garde qu'il a adressées, en 1402 semble-t-il, à l'intention des jeunes qui s'orientaient vers cette carrière contre les dérives liées à la pratique de la magie ${ }^{27}$. Parce qu'il est chanoine de Notre-Dame, il se réfugie dans les combles de sa cathédrale en 1413, au plus vif des conflits qui agitent alors la capitale, pour échapper aux représentants du parti cabochien, tandis que sa maison est mise à sac et que sa bibliothèque est anéantie ${ }^{28}$. Au-delà, les registres capitulaires confirment que Jean Durand et lui participent ensemble régulièrement aux assemblées des chanoines. En juin 1407, lors de l'une de ces réunions, le chancelier prend même la place de Durand ${ }^{29}$. En juillet 1412, ce dernier accorde à Gerson un prêt de 30 francs, pris sur le gage de la vente, réalisée par le chancelier, d'un exemplaire des Postilles de Nicolas de Lyre appartenant à la bibliothèque de Notre-Dame et pour la vente de deux manuscrits du commentaire sur les Sentences de saint Bonaventure dont il est décidé qu'ils resteront sur place ${ }^{30}$.

Les deux hommes ont aussi collaboré à l'occasion des querelles juridiques entre Notre-Dame et d'autres institutions religieuses. L'une des affaires les plus importantes a été sans doute celle du procès qui oppose les chanoines aux moines de l'abbaye de Saint-Denis en 1410 au sujet de la possession des reliques du chef du saint, lequel était honoré par les deux communautés parce qu'il était à la fois le patron de l'abbaye royale et le premier évêque de Paris. L'affaire est attestée par une documentation importante, en grande partie éditée et conservée pour l'essentiel aux

26. D. JACQUART, La Médecine..., p. 308.

27. Gerson a ainsi composé, peut-être en 1402 , le De erroribus artis magicae; à cet opuscule, ont été annexés les 28 articles contre la magie que la faculté de théologie avait publiés quelques années auparavant, en 1398, à l'époque du procès de Jean de Bar. Pour l'édition, cf. Malleus maleficarum, Paris, 1666, t. II, p. 163-173; JeAn Gerson, CEuvres complètes, éd. P. GloRieux, Paris, 1960-1973, t. X, p. 77-90 (incipit: «Collaudanti michi nuper, ut mos habet...» Aucune personnalité du milieu de Notre-Dame n'est mentionnée dans cet opuscule.) $C f$. aussi R. Veenstra, Magic and Divination at the Courts of Burgundy and France. Text and Context of Laurent's Pignon Contre les devineurs (1411), Leyde, 1998, p. 68 et 351-355 (texte de la confession de Jean de Bar d'après le ms. Paris, BnF, fr. 25552); J.-P. Boudet, «Les condamnations de la magie à Paris en 1398», Revue Mabillon, n.s., 12 (2001), p. 121-157.

28. G. Ouy, «Les premiers humanistes et leurs livres», dans A. Vernet éd., Histoire des bibliothèques françaises, t. I, p. 270.

29. Cf. Jean Gerson: «Provisores domus Dei et domini magistri Jo. Durandi, S. de Campis, M. Dangeul et Tho. de Almeto» (CEuvres complètes, t. X, p. 407); Durand est également cité à l'année 1408: cf. ibid., p. 479.

30. Document reproduit en Annexe, $n^{\circ}$ 2, d'après le ms. Paris, Arsenal, 6259 I, p. VII. À propos de l'interprétation de Bonaventure par Gerson, $c f$. P. GloRieuX, «Gerson et saint Bonaventure», dans S. Bonaventura, 1274-1974, Grottaferrata/Rome, 1974, p. 773-791. Rappelons aussi qu'un éloge de saint Bonaventure a été rédigé par Gerson le 8 novembre 1402, dans le cadre du Contra curiositatem studentium (Jean Gerson, Euvres complètes, t. III, p. 231). 
Archives nationales ${ }^{31}$. Des argumentaires de défense ayant été constitués par les deux parties, on peut en confronter les positions respectives et se faire une idée des enjeux politiques et religieux d'un conflit où, au-delà des deux institutions religieuses, s'affrontaient d'un côté la monarchie, soutien des moines de Saint-Denis, et de l'autre côté, l'évêque de Paris qui, lui, supportait logiquement les chanoines.

Le dossier de ces derniers, très détaillé et circonstancié, fait en particulier référence à plusieurs reprises aux textes produits à cette occasion par les moines de Saint-Denis, notamment aux chroniques de l'abbaye, ces récits dont étaient nées au cours du XIII siècle, par ordre du roi, les Grandes chroniques de France ${ }^{32}$. Bornons-nous à rappeler ici que c'est en 1406, plus particulièrement, que le conflit au sujet de la possession du chef de saint Denis s'est aggravé, après que le duc de Berry, collectionneur célèbre aussi bien de livres et d'objets précieux que de reliques, s'était adressé aux chanoines de Notre-Dame pour obtenir le fragment de la tête de saint Denis qu'ils prétendaient posséder, proposant en échange une tête entière de saint Philippe enfermée dans un luxueux reliquaire. C'est alors que les moines de Saint-Denis, vexés de voir un membre de la famille royale accorder autant d'importance et de prix à une pièce qu'ils estimaient fausse, en avaient appelé à Louis, duc d'Orléans. Celui-ci était donc allé faire ses dévotions à Saint-Denis, où il avait vénéré la tête qui s'y trouvait conservée et où il avait constaté par la même occasion qu'elle était entière. On aurait pu s'en tenir à ces témoignages divergents, mais l'intervention du parlement de Paris et la tenue d'un procès furent jugées nécessaires lorsque les moines décidèrent d'exposer en l'abbaye des affiches insultantes pour les chanoines; des représentants des deux parties en étaient même venus aux mains.

31. Cf. surtout Paris, AN, L 862 (Procès du chef de Saint-Denis, actes, 1409-1410); LL 362 (Mémoire dressé par les religieux de Saint-Denis à l'occasion du procès du chef de SaintDenis); LL 1326, 1 à 5 (Mémoires du chapitre de Notre-Dame à l'occasion du procès du chef de Saint-Denis). De larges extraits de ce dernier document, qui offre un état complet et détaillé des arguments présentés par les religieux de Saint-Denis aux chanoines, ont été édités par H. Delaborde, «Le procès du chef de saint Denis en 1410 », Mémoires de la Société de l'histoire de Paris et de l'Île-de-France, 11 (1884), p. 297-409. B. GuENÉE, notamment, a fait référence à ce procès à propos de l'usage médiéval des textes historiques et à la problématique des écrits authentiques ( Authentique et approuvé. Recherches sur les principes de la critique historique au Moyen Âge» [1979], réimpr. dans B. GuEnÉE, Politique et histoire au Moyen Âge. Recueil d'articles sur l'histoire politique et l'historiographie médiévales, Paris, 1981, p. 265-278). Voir aussi C. GAUVARD, «Écrire l'histoire et restaurer l'honneur au parlement de Paris. À propos de la relique du chef de saint Denis en 1410 », dans «Rerum gestarum scriptor». Histoire et historiographie au Moyen Âge. Mélanges offerts à Michel Sot, Paris, 2012, p. 509-519.

32. Ample bibliographie; pour un résumé, je me permets de renvoyer à mon étude $L a$ Bibliothèque de l'abbaye de Saint-Denis en France du IX au XVIII siècle, Paris, 1985, p. $47-$ 51. 
Si Gerson, par son envergure intellectuelle et morale, fut retenu comme avocat du chapitre de Notre-Dame, il semble que Jean Durand ait participé lui aussi activement au montage du dossier juridique et de l'argumentaire de défense des chanoines. On le voit ainsi, dès le début du mois de décembre 1406, intervenir auprès du duc de Berry, dans une délégation dont font partie plusieurs autres chanoines de la cathédrale, des étudiants et un notaire, pour recueillir de leur part une déclaration sur l'état des reliques du chef du saint tel que le duc, présent à Saint-Denis en 1398, avait pu le constater, et pour avoir la confirmation que le reliquaire conservé à Saint-Denis contenait une tête à laquelle manquait bien un morceau. Quelques jours plus tard, le 13 décembre, Jean Durand se rend avec trois de ses confrères auprès du duc de Bourgogne pour plaider la cause de NotreDame $^{33}$. Et c'est toujours Durand qui, en compagnie de Jean Voignon, un autre chanoine de Notre-Dame, ancien doyen de la faculté de médecine (en $1394)^{34}$, est chargé par l'évêque de dépouiller les livres et les registres de l'évêché afin d'y relever ce qui serait relatif au chef de saint Denis ${ }^{35}$.

Durand a compté dans son entourage immédiat au moins un autre chanoine de Notre-Dame et illustre médecin, à la carrière assez semblable à la sienne: Geoffroy Maupoivre. Lui aussi normand, mais originaire du diocèse d'Évreux, Maupoivre est, comme Durand, étudiant en deuxième année de médecine en $1379^{36}$. Devenu régent de la faculté, poste qu'il occupe jusqu'en 1389, il devient «physicien » de Jean sans Peur, pour qui il effectue des missions aussi bien à Paris qu'à Arras et à Rouen. Titulaire de plusieurs bénéfices ecclésiastiques, en particulier à Rouen et à Soissons, il obtient un canonicat à Notre-Dame en permutant avec Jean Deslandes, secrétaire du roi ${ }^{37}$. Le nom de Maupoivre revient à deux reprises dans l'inventaire après décès de Jean Durand. En 1409, il signe une reconnaissance de dette de 39 livres tournois, mentionnée dans les papiers d'archives du défunt. Maupoivre est aussi un bibliophile: nous savons qu'en 1414 il avait reçu en don de son patron, Jean sans Peur, un exemplaire du Livre des échecs

33. Paris, AN, LL 212c (Registres capitulaires de Notre-Dame); H. Delaborde, «Le procès...», p. 311.

34. À propos de ce personnage (1425), clerc du diocèse de Besançon, bachelier en médecine, maître sans doute vers 1387, régent de la faculté de médecine en 1394, chanoine de Notre-Dame de Paris, voir la notice de E. Wickersheimer, Dictionnaire..., p. 501. Il y est précisé, en particulier, que Jean Voignon fut, lui aussi, médecin de Philippe II le Hardi et qu’il participa à ses obsèques, et qu'il fut ensuite médecin de Jean sans Peur, ainsi que premier physicien de Charles VI.

35. Paris, AN, LL 212c, p. 596; H. Delaborde, «Le procès... », p. 305.

36. $C f$. H. Denifle, E. Châtelain, Chartularium..., t. III, p. 265, 449, 480; IV, p. 79; et la notice d'E. WICKERSHEIMER, Dictionnaire..., p. 180.

37. Rouen, AD de Seine-Maritime, G 1632 (détail du contenu présenté dans Inv. sommaire des archives départementales, Seine-Maritime, II, p. 31). 
moralisés, sans doute l'œuvre d'Evrard de Conty ${ }^{38}$. Durand, lui, a récupéré un autre des livres de Maupoivre, un exemplaire du Roman de la Rose; j'y reviendrai dans la dernière partie de cette étude. Mais il y a plus. Le 20 mars 1414, Maupoivre cède, avec l'agrément du chapitre, son hôtel du cloître de Notre Dame à Nicolas de Baye (1419), greffier du Parlement de Paris, ancien chanoine de Châlons devenu, lui aussi, chanoine de Paris, et auteur du célèbre journal qui relate les plus importants événements du règne de Charles VI ${ }^{39}$. Nicolas de Baye ne va rester que deux ans dans cette demeure car, en 1416, à la mort de Jean Durand, il fera l'acquisition de l'hôtel de ce dernier. La transaction est elle aussi relatée dans l'un des articles de l'inventaire après décès de notre personnage ${ }^{40}$. Il est donc temps d'examiner ce document pour essayer de reconstituer, dans la mesure du possible, le cadre de vie, les activités et les intérêts intellectuels de notre chanoine.

\section{La demeure et les activités}

Au début du $\mathrm{XV}^{\mathrm{e}}$ siècle, le cloître de Notre-Dame forme un véritable quartier ayant une vie propre. Ce quartier est fermé de toute part et on y accède par quatre portes. L'hôtel de Jean Durand, dont la disposition est soigneusement décrite dans l'inventaire, est agrémenté d'un jardin donnant sur la Seine. La porte principale, sur rue, conduit dans une salle basse qui s'ouvre sur le jardin. De là, une galerie aménagée mène à l'intérieur de la demeure, où l'on pénètre après avoir passé une autre salle basse, pièce qui était, sans doute, destinée à accueillir les visiteurs. La demeure, sur deux étages, compte vingt-six pièces, dont certaines sont luxueusement décorées et remplies de meubles, de tissus et d'objets. Il y a aussi une chapelle, avec ses parements liturgiques, un grenier, une cave, une étable, une buanderie, des chambres pour les valets ${ }^{41}$. L'inventaire de Nicolas de Baye, qui nous est aussi parvenu et qui décrit trois ans plus tard la même demeure ${ }^{42}$,

38. Cf. Mémoires de la Société archéologique d'Eure et Loir, 9 (1889), p. 466, n. 3 ; Dijon, AD de la Côte d'Or, B 388.

39. A. Tuetey, Journal de Nicolas de Baye, greffier du Parlement de Paris, 1400-1417, Paris, 1885-1888 (2 vol.); contenant aussi l'édition de son inventaire après décès de 1419, paru également en extrait: A. Tuetey, Inventaire de Nicolas de Baye, chanoine de NotreDame, greffier au Parlement de Paris sous Charles VI, précédé d'une notice biographique, s. 1., 1888. Voir aussi, sur Nicolas de Baye et son journal, A. Grün, Actes du Parlement de Paris, $1^{\text {re }}$ série: De l'an 1254 à l'an 1328, Paris, 1863-1867 (t. I, Notice sur les archives du Parlement de Paris, surtout p. L-LXV).

40. «Item, ledit hostel a esté vendu à $\mathbf{M}^{\mathrm{e}}$ Nicolas de Baye, chanoine de Paris, pour la somme de mil L fr. qui valent viiii $\mathrm{xl}$ l. p. », $c f$. Paris, AN, S 851b, p. $44 \mathrm{v}^{\circ}$. Voir aussi, pour la transaction, Paris, AN, LL 215, f. ${ }^{\circ} 34$ et 135.

41. Pour la disposition de l'hôtel de Jean Durand d'après l'inventaire, $c f$. Annexe, Document $n^{\circ} 4$.

42. Paris, AN, S 1822, n 4, document du 17 mai 1419, édité par A. Tuetey, Journal de Nicolas de Baye... 
suit une logique différente de celui de Jean Durand, car les objets y sont présentés par catégories (tapis, meubles, tissus, coffres, etc.). Mais le document indique également, pour chacun de ces objets, leur emplacement dans l'hôtel; on peut donc confronter les deux états de la demeure, à trois ans de distance et après un changement de propriétaire. Si certaines pièces ont gardé leur décor d'un propriétaire à l'autre (la chambre dite «blanche», qui est située à l'étage à côté de l' «estude» de Jean Durand, et donnant sur le jardin, étant la plus somptueuse, dans un document comme dans l'autre), d'autres révèlent que Jean Durand avait un train de vie bien plus aisé que celui de Nicolas de Baye.

Durand possède, en premier lieu, un grand nombre de bijoux, d'objets précieux, d'argenteries, auxquels s'ajoute un coffre rempli de devises. Ces pièces sont décrites au début de l'inventaire, car les exécuteurs testamentaires les ont extraites des chambres où elles se trouvaient pour procéder à leur estimation. Il en est de même des documents administratifs, d'un certain nombre d'objet de prix, de tableaux et de livres. Dans les pièces demeurent encore, en revanche, les tissus ouvragés et damassés, les tapisseries, les draps, les meubles ornés et sculptés. Dans la chapelle, Durand dispose de parements plus richement ornés que ceux qui appartiendront, trois ans plus tard, à Nicolas de Baye ${ }^{43}$. Sa cave, elle, est remplie de crus de Bourgogne et de Loire, alors qu'à l'époque de Nicolas de Baye elle ne contient plus qu'une «queue» de vin vermeil et deux «queues» en vidange... Il n'y a pas lieu de poursuivre ici une comparaison qui pourrait faire l'objet d'une recherche spécifique et qui intéresserait beaucoup, croyons-nous, les historiens des coutumes, de l'architecture, de l'artisanat et de l'art ${ }^{44}$. La différence entre les deux patrimoines s'impose en confrontant leur estimation globale, qui apparaît, dans un cas comme dans l'autre, à la fin de l'inventaire: 1.793 livres tournois, soit 1.434 livres parisis, pour Nicolas de Baye, contre 6.284 livres parisis pour Jean Durand, dont 840, soit 1.050 francs, correspondant au prix de vente des «murs » de l'hôtel.

Arrêtons-nous en revanche sur les pièces et les sections de l'inventaire de Jean Durand qui concernent de plus près sa vie intellectuelle et ses relations. La première est l'écritoire (le «scriptouere»), son bureau, qui était situé à l'étage, à côté de la chambre blanche à laquelle nous avons

43. Le contenu de la chapelle de Jean Durand, décrit p. 15 de l'inventaire, comportait un tableau de la Vierge, plusieurs vêtements liturgiques en satin, un autel de bois en forme de buffet et des chandeliers de Limoges. Plus simple en ce domaine, Nicolas de Baye avait, lui aussi, un tableau de la Vierge, mais petit et surmonté d'un chapiteau en bois d'Irlande, plus une statue, également de la Vierge, en pierre blanche et quelques autels portatifs.

44. Pour une introduction sur les aspects de la production artistique en France au début du $\mathrm{XV}^{\mathrm{e}}$ s., $c f$. au moins É. TABuret-Delahaye éd., La Création artistique en France autour de 1400, Actes du colloque international (École du Louvre, Musée des Beaux-Arts de Dijon, Université de Bourgogne), Paris, 2006. 
déjà fait référence. La seconde est l'étude, qui se trouvait juste à côté de cette pièce; mais Durand disposait en plus d'une autre petite étude ouvrant sur la terrasse de l'hôtel. C'est dans ces deux locaux que Durand conservait ses livres de son vivant, de même, sans doute, qu'une partie des tableaux et les instruments scientifiques qui sont décrits à la fin du document.

Si l'inventaire ne signale plus que la présence, dans l'écritoire, d'une armoire de bois d'Irlande, d'un coffre de noyer et de quelques effets personnels (des gants et une ceinture ouvragée d'argent), cette pièce a joué un rôle important non seulement pour l'établissement de la documentation personnelle de Jean, mais aussi parce c'est sans doute dans son cadre que se sont déroulées quelques-unes des opérations du mécénat bibliophilique de Philippe II le Hardi. En effet, les comptes de l'hôtel de Bourgogne nous apprennent qu'en 1402, pendant tout un mois, du 25 février au 30 mars, le duc a séjourné chez Jean Durand. Il revient le voir quelques mois plus tard, à deux reprises, le 15 août et le 7 septembre, et s'arrête dîner et dormir chez lui ${ }^{45}$. Deux ans à peine avant le décès du duc de Bourgogne, qui surviendra en avril 1404, il nous faut essayer de préciser les circonstances de ces séjours répétés et d'éclairer les relations que les deux hommes ont alors entretenues, des relations probablement plus riches et complexes que celles qu'un malade entretient avec son médecin.

C'est le 9 février 1402, en effet, que Philippe avait fait engager deux jeunes enlumineurs, Poloquin et Jeannequin Malouel (on reconnaîtra les célèbres frères Limbourg) pour qu'ils décorent une Bible. Ce travail devait être réalisé en l'hôtel de Jean Durand, qui avait reçu 600 francs pour rétribuer les deux artistes. Le chanoine était chargé de suivre la progression de leur entreprise et de contrôler qu'ils ne se consacrent à aucune autre commande ${ }^{46}$. Le projet devait commencer le 6 mars et les deux frères devaient recevoir un salaire de 10 sous parisis «pour chaque jour ouvrable et non ouvrable», et ce pendant une période de quatre ans. P. de Winter a fait remarquer que ce salaire, correspondant à 7,5 gros, était assez élevé puisque l'oncle des deux

45. P. DE Winter, La Bibliothèque..., p. 107, d'après E. Petrt, Itinéraires de Philippe le Hardi et de Jean sans Peur, ducs de Bourgogne (1363-1419) d'après les comptes de dépenses de leur hôtel, Paris, 1888, p. 322 et 328-329.

46. $C f$. l'extrait suivant: «pour parfaire les histoires d'une belle et notable Bible que avoit nagaires fait commencer ledit seigneur, que pour l'accomplissement d'icelle et des ystoires qui devront y estre faites, iceux Polequin et Jeannequin ne se pouvoient louer a autre qu'a mondit seigneur, mais entendre et besoigner seulement en l'ouvrage d'icelle », d'après le document Dijon, AD de la Côte d’Or, B 1526, fo 292v-293 (compte de Jean Chousat): P. DE Winter, La Bibliothèque..., p. 107 et 265 ; également cité par G. PEIGNOT, De l'ancienne bibliothèque des ducs de Bourgogne, Dijon, 1841. Sur la production et le commerce des manuscrits à Paris et l'activité des ateliers d'enlumineurs dans le cloître de Notre-Dame, $c f$., outre les travaux de P. de Winter, R. H. et M. A. Rouse, “Illitterati et uxorati”. Manuscripts and their Makers: Commercial Book Producers in Medieval Paris, 1200-1400, Londres, 2000 . 
Limbourg, Jean Malouel, recevait à la même époque 12 gros par jour, et que le sculpteur Claus Sluter en recevait 8. Les deux jeunes frères étaient donc déjà célèbres et recherchés. Le financement du projet avait été en partie assuré par le marchand et banquier italien Giacomo Rapondi, qui résidait alors à Paris ${ }^{47}$.

En janvier 1403, à l'occasion du nouvel an, le duc fit remettre à chacun des deux artistes une gratification de 10écus, pour qu'ils avancent dans leur travail $^{48}$. Mais plus d'un an plus tard, en 1404, la Bible n'était toujours pas finie. Philippe le Hardi étant mort en avril 1404, le contrat fut rompu et les frères Limbourg passèrent au service du duc de Berry ${ }^{49}$. Trois ans plus tard, en 1407, Jean Durand contresigna un mandement de Jean sans Peur pour qu'une somme de 60 francs, allouée elle aussi par Giacomo Rapondi trois ans plus tôt, en 1404, fût bien versée à trois autres enlumineurs, Jacques Coene, Ymbert Scanier et Hainselin de Haguenau, pour réaliser une autre Bible enluminée, en latin et en français. Selon l'hypothèse de Patrick de Winter, il s'agissait là de finaliser la commande laissée inachevée par les Limbourg ${ }^{50}$.

Jean Durand a aussi possédé quelques instruments d'astronomie et de mathématiques. On dénombre quatre astrolabes, dont trois en laiton et un en bois; un «tableau rond» en parchemin où étaient figurés les douze signes; un coffret peint avec les signes zodiacaux et les mouvements du soleil et de la lune ${ }^{51}$; un «tableau rond ouquel est la calculacion a doubler les poins de l'escequier par grains de fourment», soit un instrument d'arithmétique ${ }^{52}$. Ils sont décrits à la fin de l'inventaire, en même temps que quelques objets d'art, quelques tableaux (dont l'un était un portrait du duc de Bourgogne) et deux cartes (des «mappemondes ») en parchemin, dont l'une décorée des armoiries du même. Les instruments astronomiques renvoient au rôle de conseiller et d'astrologue que le médecin a joué auprès du duc de Bourgogne et de la famille royale, tout comme à la charge qui lui avait été confiée par le

47. P. De Winter, La Bibliothèque..., p. 67. Sur les Rapondi, marchands, banquiers et bibliophiles à Paris, $c f$. B. LAMBERT, The City, the Duke and their Banker : the Rapondi Family and the Formation of the Burgundian State (1384-1430), Turnhout, 2006; B. Buettner, «Jacques Raponde, marchand de manuscrits enluminés », Médiévales, 14 (1988), p. 23-32.

48. Dijon, AD de la Côte d'Or, B 1532, fo $194 \mathrm{v}^{\circ}-195 \mathrm{r}^{\circ}$; P. DE WINTER, La Bibliothèque..., p. 266.

49. Ibid., p. 266.

50. Dijon, AD de la Côte d'Or, B 1547, $\mathrm{f}^{\circ} 140 \mathrm{v}^{\circ}$, cité par P. DE WinTER, La Bibliothèque..., p. 266.

51. À propos des astrolabes et autres instruments astronomiques, $c f$. E. Poulle, Les Instruments astronomiques au Moyen Âge, Paris, 1983; ID., Les Sources astronomiques: textes, tables et instruments, Turnhout, 1981 (Typologie des sources du Moyen Âge occidental, 39).

52. On pense au Compte des LXIIII poins de l'escequier double que Robert du Herlin, secrétaire de Charles VIII, prétend avoir traduit de latin en français dans le ms. Paris, BnF, fr. $2000, \mathrm{f}^{\circ} 51 \mathrm{r}^{\circ}-55 \mathrm{r}^{\circ}$, daté de 1493 . 
roi en 1379. On a bien conservé, à la bibliothèque du Saint John's College d'Oxford, un manuscrit d'œuvres d'astronomie et d'astrologie provenant de la bibliothèque de Charles V et de Charles VI où figurent, sur les gardes du début, les horoscopes de naissance de Marguerite de Flandre, duchesse de Bourgogne, et de son fils Antoine de Bourgogne, né en 1384 (Oxford, Saint John's College, ms. 164, fo [IIv]-[III]), et il se peut, comme l'a suggéré J.-P. Boudet, que ces carrés astrologiques soient l'œuvre de Jean Durand. Cependant, à défaut de sources plus explicites, cette hypothèse ne peut être vérifiée. Par ailleurs, aucun autre spécimen de ce qui pourrait être l'écriture de Jean Durand ne semble avoir été conservé ${ }^{53}$.

\section{Les livres d'un médecin de cour}

Du vivant de Jean Durand, comme on l'a rappelé plus haut, les livres devaient être répartis dans les deux études aménagées en sa demeure mais, au moment de l'inventaire, ces deux pièces ne contenaient plus que quelques meubles, des tables et des fauteuils, ainsi que des coffres remplis de tissus. Cependant, l'étude principale, décorée d'un tapis, d'une tapisserie de haute lice et d'un autel portatif, semble avoir gardé un aspect de pièce d'apparat. Mais avec 35 volumes, dont la valeur est évaluée à 94 livres, 16 sous parisis, la bibliothèque de Jean Durand est somme toute une collection modeste, surtout si on la compare avec celle de son successeur dans le même hôtel, Nicolas de Baye, possesseur de 198 volumes évalués à 430 livres tournois à sa mort en 1419, ce qui représentait une valeur de près du quart de ses biens meubles ${ }^{54}$. Cette quantité reste exceptionnelle même si on tient compte du fait que, sur les 198 volumes que Nicolas de Baye possédait à sa mort, une vingtaine lui avait été laissée en gage par des personnes de son entourage, surtout des chanoines de Notre-Dame et leurs familiers. Il n'est pas impossible que Nicolas, actif au Parlement au plus vif des conflits entre Armagnacs et Bourguignons, ait pu également recueillir des livres issus de collections privées de morts ou d'exilés, mais aucun article de l'inventaire ne permet de l'affirmer avec certitude et on n'a conservé aucun des manuscrits qui lui ont appartenu.

53. J.-P. BoudeT, «Charles V, Gervais Chrétien... », p. 23. Le ms. d'Oxford contient, en outre, l'Espere en françois de Nicole Oresme, le Livre des elections et le traité de l'astrolabe de Pèlerin de Prusse, une traduction française du Liber introductorius d'Alcabitius, et enfin les horoscopes de Charles V et de ses enfants. Pour ces horoscopes, $c f$. E. Poulle, «Horoscopes princiers des $\mathrm{XIV}^{\mathrm{e}}$ et $\mathrm{XV}^{\mathrm{e}}$ siècles », Bulletin de la Société nationale des antiquaires de France, séance du 12 février 1969, p. 63-77 (p. 63-69), réimpr dans ID., Astronomie planétaire du Moyen Âge latin, Aldershot 1996, art. n VIII.

54. Pour une appréciation de la bibliothèque de Nicolas de Baye par rapport à celle de ses collègues parlementaires, $c f$. F. AutRAND, «Culture et mentalité. Les librairies des gens du Parlement au temps de Charles VI», Annales E.S.C., 28 (1973), p. 1219-1244. 
Si nous considérons à présent les bibliothèques d'autres médecins contemporains, il ne reste, pour la France, qu'une petite quinzaine d'inventaires décrivant comme dans ce cas des collections «complètes». Or, celle de Jean Durand paraît bien moins importante que les bibliothèques de ses homologues: Arnoul de Halle, qui fut aussi chanoine de Cambrai et qui possédait 73 volumes en $1427^{55}$, ou Astruc del Sestrier, médecin d'Aix-enProvence, qui en 1439 était, lui, propriétaire de 179 volumes ${ }^{56}$. Plus proche du nôtre, géographiquement et chronologiquement, Pierre Cardonnel, lui aussi chanoine de Notre-Dame et médecin, formé à la faculté de Paris, possédait, quelque quinze ans plus tard, une bibliothèque à peu près de la même importance, avec 32 volumes $^{57}$. À la fin du siècle, Simon de Phares a certainement possédé une bibliothèque très importante, mais elle a été dispersée; une trentaine de volumes seulement ont pu lui être attribués avec certitude $^{58}$.

Mais la collection de Jean Durand se démarque nettement de celles de ses confrères par son contenu. En effet, la médecine (une discipline dont relèvent presque tous les livres d'un Pierre Cardonnel, par exemple) n'est ici représentée que par une dizaine de manuscrits. À l'exception du Canon d'Avicenne et d'un «comment de medicine» dont l'auteur n'est pas précisé, prévalent les textes d'orientation «pratique», comme la Practica de Serapion et celle de Guillaume de Saliceto. Il y a encore un livre des fièvres et un livre génériquement défini de medicina, qu'il est impossible d'identifier. Durand possédait aussi un exemplaire du Defensorium vite. Un traité portant ce titre a été attribué à Arnaud de Villeneuve $(1311)^{59}$, mais il s'agit évidemment d'un apocryphe, non répertorié parmi les œuvres

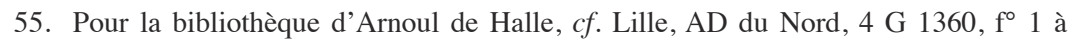
40; $\mathrm{f}^{\circ} 20$ à 27 pour les livres (manquent les $\mathrm{f}^{\circ} 20$ à 26 ); $\mathrm{f}^{\circ} 34$ pour la recette des livres; édition du document par L. de LABORDE, Les Ducs de Bourgogne au XVe siècle. Les lettres, les arts et l'industrie pendant le XVe siècle, 1849-1852, Paris, t. II, p. 400-413 (seulement pour l'inventaire des livres, actuellement mutilé; n'a pas relevé la partie concernant la recette des livres).

56. Aix-en-Provence, AD des Bouches-du-Rhône, 306 E/266, f $\mathrm{f}^{\circ}$ 4-13 (les livres sont décrits aux $\left.\mathrm{f}^{\circ} 6-9 \mathrm{v}^{\circ}\right)$; la bibliothèque d'Astruc del Sestrier a été étudiée par D. IANCU-AGOU, «L'inventaire de la bibliothèque et du mobilier d'un médecin juif d'Aix-en-Provence au milieu du Xve siècle», Revue des études juives, 134 (1975), p. 47-80 (avec un commentaire détaillé et une traduction du document; édition p. 48 à 52).

57. Pour la bibliothèque de Pierre Cardonnel, dont l'inventaire (1438) est conservé sous la même cote que celui de Jean Durand (S 851B, au n ${ }^{\circ} 17$ ), $c f$. A. ChérEAu, «La bibliothèque d'un médecin au commencement du $\mathrm{Xv}^{\mathrm{e}}$ siècle», Bulletin du bibliophile, 1863, p. 224-243, paru à part sous le titre La Bibliothèque d'un médecin au commencement du XV siècle, Paris, 1864, p. 14-22 (pour la partie concernant les livres); F. Autrand, «Culture et mentalité...», p. 1226.

58. Pour la reconstitution de la bibliothèque de Simon Phares, $c f$. J.-P. Boudet, Lire dans le ciel. La bibliothèque de Simon Phares, Bruxelles 1994 (Les publications de Scriptorium, $10)$.

59. E. WiCKERSHEIMER, Dictionnaire..., p. 48. 
faussement attribuées au maître catalan par J.A. Paniagua ${ }^{60}$. Notons aussi la présence de quelques traités de chirurgie, et en particulier celui de Teodorico Borgognoni (1296).

Parmi les ouvrages d'astronomie et d'astrologie se distinguent quelques textes importants, comme l'Almageste de Ptolémée, dans un exemplaire de prix, estimé à 8 livres, ainsi qu'un mystérieux «livre d'astronomie nommé Hermes » et deux exemplaires des tables alphonsines. Dans un domaine connexe à l'astronomia mais destiné à l' «esbatement» des laïcs, Jean Durand a possédé un «livre des jeux de fortune» anonyme (incipit du $2^{\mathrm{e}}$ folio: «Comme je dis »), qui pourrait correspondre à l'un des traités de divination par les sorts en moyen français récemment étudiés par Solange Lemaitre Provost ${ }^{61}$. Il n'est pas aisé, à défaut de précisions supplémentaires, d'identifier le sermon de Nicolas Oresme mentionné dans l'inventaire («Item, le sermon $\mathrm{M}^{\mathrm{e}}$ Nicolas Oresme»). La formation reçue par Jean Durand en tant que maître ès arts est confirmée par la présence, dans sa collection, du Doctrinal d'Alexandre de Villedieu et du Grécisme d'Évrard de Béthune. Le Dilucidaire, anonyme, ne semble pas correspondre au Lucidarium d'Honorius Augustodunensis, recueil raisonné d'auteurs ecclésiastiques composé à la fin du XII siècle, car le mot «canones », cité dans l'inventaire, ne semble pas y figurer.

Moins attendus dans la bibliothèque d'un médecin, quelques titres significatifs renvoient au goût de Jean Durand pour la littérature allégorique et morale en français. Outre deux traductions (celles de la Consolation de Boèce et des Métamorphoses d'Ovide), le chanoine possède un exemplaire du Roman de la Rose qui lui venait de son confrère Geoffroy Maupoivre, le médecin de Jean sans Peur dont on a parlé plus haut. Rappelons que cet ouvrage, largement apprécié dans le milieu des chanceliers royaux aux $\mathrm{XIV}^{\mathrm{e}}$ et XV ${ }^{\mathrm{e}}$ siècles ${ }^{62}$, fut, au cours de l'été 1402 à Paris, au cœur d'une querelle littéraire entre Christine de Pizan et l'humaniste Jean de Montreuil. Deux illustres membres du chapitre de Notre-Dame y furent aussi impliqués: Pierre Col, frère de l'humaniste Gontier, et Jean Gerson, dont nous avons évoqué plus haut, à propos de l'affaire des reliques du chef de saint Denis,

60. J. A. Paniagua, Studia arnaldiana. Trabajos en torno a la obra medica de Arnau de Vilanova, ca 1240-1311, Barcelone, 1995.

61. S. Lemaitre-Provost, Les Livres de sort en moyen français. Étude et édition critique, thèse de 1'université Laval, Québec, dirigée par C. Baker, 2010. L'épouse de Philippe le Hardi, Marguerite de Flandres, possédait plusieurs ouvrages de cette sorte: voir P. DE WINTER, La Bibliothèque..., p. 170-172.

62. P.-Y. BADEL, Le Roman de la Rose au XIve siècle. Étude de la réception de l'œuvre, Genève, 1980, p. 165-179; S. Нuот, The Romance of the Rose and its Medieval Readers: Interpretation, Reception, Manuscript Transmission, Cambridge, 1993. Sur la réception du texte et l'iconographie des manuscrits, $c f$. aussi N. Coilly et M.-H. Tesnière éd., L'Art d'aimer au Moyen Âge. Le Roman de la rose [catalogue de l'exposition de la BnF], Paris, 2012. 
les rapports de collaboration qu'il a entretenus avec Jean Durand ${ }^{63}$. Le chancelier, qui composa le 18 mai 1402 un traité contre le Roman de la Rose, a aussi dédié plusieurs sermons à ce sujet ${ }^{64}$, mais le nom de Durand ne revient dans aucun de ces écrits, pas plus qu'il n'est cité, à ma connaissance, dans les lettres et les textes que les autres auteurs intervenant dans cette dispute littéraire ont alors composés.

Parmi les titres les plus intéressants de cet inventaire figurent les Problemata en français, en 41 cahiers de parchemin non reliés, que les priseurs estiment néanmoins à 8 livres. Ce texte pourrait correspondre à la traduction de l'ouvrage pseudo-aristotelicien commenté par Pietro d'Abano qu'Évrart de Conty, médecin de Charles V, avait réalisée en 1380 pour le compte du roi et dont il avait également légué un exemplaire en 1403, pour son jubilé, à la bibliothèque de la faculté de médecine ${ }^{65}$. Dans cette hypothèse, on pourrait aussi rattacher au même Évrart de Conty l'exemplaire du Livre des échecs moralisés qui est également cité, comme texte anonyme, dans l'inventaire de Jean Durand ${ }^{66}$.

Il faut consacrer encore quelques mots aux manuscrits des chroniques: outre l'exemplaire des Chroniques de France, deuxième titre cité dans l'inventaire, on remarque en particulier la présence de l' «epistre de Gilles de Pontoise de la vie de saint Denis ». Il s'agit d'un exemplaire des chroniques de Saint-Denis qui avaient été présentées par Gilles de Pontoise, abbé de Saint-Denis, au roi Philippe le Long. Ce texte, avec d'autres chroniques de l'abbaye, avait été cité à l'occasion du procès de 1410 à propos de la possession des reliques du chef de saint Denis auquel nous avons fait référence plus haut, un procès dont la documentation, du côté du chapitre de Notre-Dame, avait été rassemblée par Jean Durand ${ }^{67}$.

63. Christine de Pizan, Jean Gerson, Jean de Montreuil, Gontier et Pierre Col, Le Débat sur le Roman de la Rose, éd. E. Hicks, Paris, 1977.

64. Jean Gerson, Euvres complètes, t. I, p. 119.

65. La suggestion a été formulée par J.-P. Boudet. Pour le legs par Évrart de Conty des Problemata (et d'autres livres) à la faculté de médecine, $c f$. E. Wickersheimer, Commentaires de la Faculté de médecine de l'Université de Paris, t. I, Paris, 1915, p. 32; voir aussi p. 65.

66. Évrart de Conty, Le Livre des eschez amoureux moralisés, éd. F. Guichard-Tesson et B. Roy, Montréal, 1993. Nous avons fait référence plus haut à l'exemplaire de cette œuvre que le duc de Bourgogne avait reçu de Geoffroi Maupoivre, médecin de Jean sans Peur, en 1414 de Philippe Jossequin, gardien des joyaux du roi de France.

67. Trois exemplaires de ce texte sont conservés à la $\mathrm{BnF}$, mais aucun ne semble avoir appartenu à Durand. Il s'agit des mss. Paris, BnF, fr. 2090-2092, qui constituent sans doute l'exemplaire original offert par Gilles de Pontoise au roi, et des mss. Paris, BnF, lat. 5286 et 13836. Ce dernier, troisième recueil d'une série initialement composée de trois volumes, a été copié par Guillaume Lescot et vient donc très vraisemblablement aussi de l'abbaye de Saint-Denis. Cf. L. Deliste, Comptes rendus de séances de l'Académie des inscriptions et belles-lettres, 5 (1861), p. 247-249. 
En guise de bilan, la bibliothèque de ce proche de la famille royale, si elle n'a pas été très importante, est pourtant digne d'un homme de cour. Physicien du duc Philippe le Hardi, Durand en a soutenu le mécénat bibliophilique, à l'époque où le cloître de Notre-Dame est un lieu de référence pour les artistes et les enlumineurs parisiens. Attentif aux questions d'actualité et engagé, pour le compte de la cathédrale, dans les affaires juridiques et morales qui agitent son époque, le chanoine a également possédé des ouvrages d'histoire, de philosophie et de littérature allégorique en langue vernaculaire. Ces intérêts littéraires renvoient aux relations prestigieuses qu'il a entretenues dans les milieux de l'université et de la cour: nous avons mentionné Jean Gerson, Jean Voignon, Geoffroy Maupoivre, Louis de Bar et même aperçu, sans doute, la personnalité majeure d'Évrart de Conty. Mais ces lectures témoignent aussi de l'importance que les arts libéraux ont tenu dans la constitution du profil culturel de Jean Durand. De sa formation en ces disciplines découle aussi l'intérêt qu'il a porté à l'astronomie et à l'astrologie. Spécialiste reconnu en ces domaines, Durand semble même les avoir préférés à la médecine, une discipline qu'il a pratiquée mais dont il n'a sans doute pas approfondi les aspects théoriques et spéculatifs.

Donatella Nebbiai - Institut de Recherche et d'Histoire des Textes (CNRSUPR 841), Paris.

\section{Les livres de Jean Durand ( $† 1416)$, "physicien » et astronome}

Jean Durand, chanoine de Notre-Dame, fut médecin et astronome. Titulaire de la première bourse pour l'enseignement de l'astrologie à Paris sous Charles V, il fut le "physicien» de Philippe le Hardi, duc de Bourgogne. Un inventaire après décès de ses biens, découvert aux Archives nationales ( $S$ $851 \mathrm{~B}, \mathrm{n}^{\circ} 4$ ), permet de reconstituer sa biographie et son cadre de vie. La liste des livres ouvre la perspective d'un milieu raffiné qui, au-delà des intérêts professionnels, cultive la littérature en langue vernaculaire et apprécie la culture historique.

Astrologie - astronomie - bibliothèque - collège - enlumineur - manuscrit - médecine

\section{Jean Durand's Books ( $†$ 1416), " Physician " and Astronomer}

Jean Durand, canon of Notre-Dame, was a physician and an astronomer. Physician of Philippe le Hardi, duke of Burgundy, he was also the first recipient of a fellowship dedicated to the teaching of astrology by Charles the Fifth in Paris. A post-mortem inventory of his personal belongings recently discovered at the Archives nationales (S851B, $\left.n^{\circ} 4\right)$, permits to reconstitute his biography and living environment. Beyond the professional interests, his list of books does open the perspective of the sophisticated environment of the King's court, promoting the literature in vernacular language and historical culture.

Astrology - astronomy - college - illuminator - library - manuscript medicine 


\section{Annexes}

\section{Document 1}

\section{Extraits de l'inventaire du trésor de la cathédrale de Châlons, mentionnant Jean Durand}

1410-1413. Inventaire de la cathédrale de Saint-Étienne de Châlons-en-Champagne. Reims, AD Marne, fonds du chapitre Saint-Étienne de Châlons, G 454 (olim arm. 1, liasse 53), $\mathrm{n}^{\circ} 10$.

Édition: M. PÉLICIER, Inventaire des meubles et joyaux de la cathédrale de Châlons en 1410, Paris, 1886, 56p. (extrait du Bulletin archéologique du comité des travaux historiques et scientifiques, 2, 1886).

Bibliographie: A. Genevois, J.-F. Genest, A. Chalandon, Bibliothèques de manuscrits médiévaux en France, Paris, 1987, n 368.

Les pages indiquées renvoient à l'édition de M. PéLICIER, $c f$. supra:

p. 25 : «113 (A). Item, unus tassellus rotundus de panno aureo damasco livido cum duobus esguilletis de serico munitis argento, ymaginatus ad sanctum Stephanum de brodeura cum lapidantibus, et in circumferentia plenus perlis de computo, et circa ymaginaturam aliis perlis de computo necnon minutis aliis, estque de cappa data per magistrum Johannem Durandi, quondam archidiaconum Cathalaunensem, qui est in scrinio rotundo de corio.»

p. 27: «149. Item, duo auricularia de sendali rubeo quorum unum grossius est altero, que venerunt de bonis magistri Johannis Durandi. »

p. 35 : «232. Item, alia cappa data per dominum Johannem Durandi, archidiaconum Cathalunensem, de velveto rubeo seminato branchis bauchie ${ }^{68}$. »

\section{Document 2}

\section{Extraits des registres du chapitre de Notre-Dame de Paris, 1412}

1412,11 juillet.

Jean Gerson remet à Jean Durand la somme de 30 francs, en gage de la vente d'un livre de la bibliothèque de l'église, et pour payer deux autres livres qui devront être conservés dans la bibliothèque.

Paris, Bibl. de l'Arsenal ms. 6259 (I), p. VII.

Éd. A. Franklin, Les Anciennes Bibliothèques de Paris, Paris, 1867, t. I, p. 49-50.

xi julii 1412.

Hodie magister Joannes de Gersonio, cancellarium hujus ecclesie, tradidit triginta francos magistri Joanni Durandi in praesentia et de consensu capituli per modum mutui recipiendi super pecunia venditionis Postilla de Lyra quae est de libraria ejusdem ecclesiae et pro solvendo duo volumina Bonaventurae super Sententias, quod reservetur in eadem libraria fieri deliberatum fuit sabbato $5^{\mathrm{a}}$ et ultima capituli generalis beati Joannis Baptiste.

68. Bauchia, à traduire par «persil». 


\section{Document 3}

\section{Extraits de l'inventaire après décès de Jean Durand, 1416}

Dossier d'exécution testamentaire de Jean Durand, chanoine de Paris, décédé le 3 juillet 1416 (Paris, AN, S $851 \mathrm{~B}, \mathrm{n}^{\circ} 4$ )

[p. 1] Ensuit l'inventoire des biens appartenans a l'execucion feu $\mathrm{M}^{\mathrm{e}} \mathrm{Jehan}$ Durant, a son vivant chanoine de l'eglise de Paris, qui trespassa en son hostel au cloistre de la dicte eglise l'an mil cccc et xvi, le vendredi tiers jour du moys de juillet, fait presens les executeurs dudit deffunct et seigneur de ladicte eglise cy dessoubs nommez et par les journees cy aprés declairees lesquels furent prisez, est assavoir la vaysselle d'argent per Guillaume Pastoureau, orfeuvre et franc sergent de ladicte eglise et les aultres biens par Jehan de Solante, priseur juré en la ville de Paris, et inventoriez par Nicolas Lesellier, prestre tabellion d'icelle.

[...] [p. 36]

Ensuivent les livres prisiez et trouvez oudit hostel:

Le mercredi $\mathrm{xv}^{\mathrm{e}}$ jour dudit moys, presens les executeurs et le frere et neveu dessusdit, maistre Olivier de Lempire, libraire juré en l'université de Paris, prisa les livres qui ensuivent:

Premierement, $1^{\mathrm{e}}$ petite Bible de menue lectre, commençant ou second fueillet hoc doctus prelato, prisiee

xi 1.

Item, unes croniques de France, commençant ou second fueillet Comment Charles, prisié

Item, 1 breviaire en deux volumes a l'uzage de Rouen, le greigneur commençant ou second fueillet et in tertio et le petit volume ou second fueillet fieri amen. Prisié

xii 1.

Item, 1 Avicenne en deux volumes ${ }^{69}$, l'un commençant ou second fueillet cap $^{m} x v i i$ et l'autre ou ii fueillet capitula quinti in rubro, prisié

viii 1.

Item, la Guillermine, commençant ou ii fueillet a multo $^{70}$. Prisié

Item, le Dilucidaire ${ }^{71}$, commençant ou ii fueillet canones

vi 1 .

Item, 1 livre d'astronomie nommé Almageste ${ }^{72}$, commencant ou ii fueillet et eos. Prisié

viii 1.

69. Avicenne, Canon.

70. Guillaume de Saliceto, Practica seu summa conservationis sanitatis

71. Il est difficile d'identifier ce titre avec un exemplaire du Lucidarium d'Honorius Augustodunensis, manuel pour la formation des prêtres composé de trois livres, car le mot «canones», mentionné dans l'inventaire, ne figure pas dans ce traité. Sur les Lucidaires, très répandus au Moyen Âge et en particulier aux $\mathrm{XII}^{\mathrm{e}}$, $\mathrm{XIII}^{\mathrm{e}}$ et XIV ${ }^{\mathrm{e}}$ s., $c f$. Y. LefÈvRE, L'Elucidarium et les Lucidaires. Contribution, par l'histoire d'un texte, à l'histoire des croyances religieuses en France au Moyen Age, Paris, 1954.

72. Ptolémée, Almagestum, œuvre transmise par plusieurs traductions latines (notamment par celle, arabo-latine, de Gérard de Crémone, et par une traduction gréco-latine effectuée en Sicile au milieu du XII ${ }^{\mathrm{e}} \mathrm{s}$.). 
Item, un aultre livre d'astronomie nommé Hermes ${ }^{73}$, commençant ou ii fueillet et venena letalia, prisié

xxiiii s.

Item, un aultre livre d'astrologie commençant ou ii fueillet tannis, prisié

xxiiii s.

Item, le Livre des eschés moralizé en françoys ${ }^{74}$, commençant ou ii fueillet et sont fueilles, prisié

iiii 1.

Item, 1 Doctrinal ${ }^{75}$ glosé, commençant ou ii fueillet cens hec vox, prisé

vi 1.

Item, 1 vieil Romant de la rose que on dit estre a m ${ }^{\mathrm{e}}$ Gieffroy Manpoyvre, commençant ou ii fueillet rechignié, prisié

xxiiii s.

Item, 1 livre des jeux de fortune ${ }^{76}$, commençant ou ii fueillet Comme je $d y$, prisié

xxxii s.

Item, 1 commant de medicine, commençant ou ii fueillet tate, prisié

$\mathrm{XX} \mathrm{S.}$

Item, 1 livre qui contient au commencement une epistre de l'abbé Gile de la vie saint Denis ${ }^{77}$, commençant ou ii fueillet ignem zelumque, prisié

xxiiii s

Item, Boece De consolacion en françoys, commencant ou ii fueillet quant voyt sont peuple, prisié

xx s. [p. 38]

Item, la Cyrurgie $\mathrm{m}^{\mathrm{e}}$ Thierry $^{78}$, commençant ou ii fueillet si ultra, prisié

X S.

Item, 1 livre de medecine qui se commence Liber morborum commençant ou ii fueillet vel unctuosus, prisié

viii s.

73. Hermès Trismegiste, auteur présumé de divers traités astrologiques et de magie astrale, $c f$. P. Lucentini, V. Perrone Compagni, I Testi e i codici di Ermete nel Medioevo, Florence, 2001.

74. Évrart de Conty, Le Livre des eschez amoureux moralisés, éd. F. Guichard-Tesson et B. Roy, Montréal (Bibliothèque du Moyen Français, 2), 1993. Il pourrait aussi s'agir d'une version française du Ludus scaccorum, œuvre allégorique et morale de Jacques de Cessoles ( $c f$. par exemple la version de Guillaume de Saint-André : J.-M. CAuneau, Le Jeu des échecs moralisés de Guillaume de Saint-André, Rennes, 1996), ou celle de Jean de Vignay, actif entre 1326 et 1350, qui fut dédiée au duc de Normandie ( $c f$. Dictionnaire des Lettres Françaises, Le Moyen Âge, éd. G. Hasenohr, M. Zink, Paris, 1992, p. 730).

75. Alexandre de Villedieu, Doctrinal.

76. Probablement un livre de sorts. Voir à titre d'exemple le troisième texte contenu dans le ms. Paris, BnF, lat. 7352, du début du Xve s., mentionné sous le titre de Gieu de fortune ou de Roüe de fortune.

77. Yves de SAINT-Denis, Vie de saint Denis suivie d'un abrégé de l'histoire de France, recueil composé à Saint-Denis sous le gouvernement de Gilles de Pontoise (1326) et dédié à Philippe V, dit le Long; $c f$. La Librairie de Charles V, Paris, 1968, p. 78; L. DelisLe, Comptes rendus des séances de l'Académie des Inscriptions et Belles Lettres, 5 (1861), p. 247-249. La présence de ce texte parmi les livres de Jean Durand est logique dans la mesure où il fut utilisé par l'auteur du mémoire de Notre-Dame lors du procès du chef de saint Denis : voir H. Delaborde, «Le procès... », p. 352-353, 375, 397-398 et 401.

78. Teodorico Borgognoni ou de Cervia, fin du XIII ${ }^{\mathrm{e}} \mathrm{s}$. 
Item, 1 livre de cirurgie, commençant ou ii fueillet aparante

xii s.

Item, les tables d'Alfons ${ }^{79}$, commençans ou ii fueillet martius

iiii s.

Item, unes gloses sur Avicene, commençans ou ii fueillet de cefalea, prisié

xii s.

Item, le livre des fieuvres, commençant ou ii fueillet ab eo permanet, prisié

iiii s.

Item, 1 livre d'astronomie, commençant ou ii fueillet magisterii, prisié

viii s.

Item, Metamorphoseos ${ }^{80}$, commençant ou ii fueillet sic modo

vi s.

Item, les tables d'Alfonse, commençans ou ii fueillet sciatur

iiii s.

Item, le livre de Serapion ${ }^{81}$, commençant ou ii fueillet dolorem

viii s.

Item, le sermon $\mathrm{m}^{\mathrm{e}} \mathrm{N}$. Oresme ${ }^{82}$, commençant ou ii fueillet eius frequenter, prisié

ii s.

Item, uns Synodaulx, commençans ou ii fueillet etas

ii s.

Item, 1 livre de cirurgie, commençant ou ii fueillet caste, prisié

ii s.

Item, 1 Gressisme ${ }^{83}$ glosé, commençant ou ii fueillet et sit

ii s.

Item, 1 livre en papier nommé Deffensorium vite ${ }^{84}$, commençant ou ii fueillet quemadmodum, prisié

iiii s.

Item, 1 livre de medecine en papier, commençant ou ii fueillet significabat, prisié

iiii s.

Item, xli quayers en parchemin escrips et contenant Problemata en françoys ${ }^{85}$, prisiés

viii 1.

79. Tables astronomiques attribuées à Alphonse, roi de Castille (1284), mais dont la version latine a été réalisée à Paris dans les années 1320; cf. E. Poulle, Les Tables alphonsines, avec les canons de Jean de Saxe, Paris, 1984; ID., «Les astronomes parisiens au XIV $v^{\mathrm{e}}$ siècle et l'astronomie alphonsine», Histoire littéraire de la France, t. XLIII, fasc. 1, Paris, 2005, p. 1-54.

80. Ovide, Métamorphoses.

81. Jean Serapion, auteur d'une Practica medicinae ( $\left.\mathrm{IX}^{\mathrm{e}} \mathrm{s}.\right)$.

82. Identification difficile; peut-être pourrait-il s'agir du Contra astronomos judiciarios de cet auteur, qui a aussi circulé sous le titre de Sermo? Cf. A. Menut, «A Provisional Bibliography of Oresme's Writings. A Supplementary Note», Medieval Studies, 31 (1969), p. 288.

83. Évrard de Béthune, Graecismus. I p. [Ps.-]Arnaud de Villeneuve, Defensorium vite: $c f$. L. Thorndike, P. Kibre, A Catalogue of Incipits of Medieval Scientific Writings in Latin, Londres, 1963, col. 1185.

84. [Ps.-]Arnaud de Villeneuve, Defensorium vite: $c f$. L. Thorndike, P. Kibre, A Catalogue of Incipits of Medieval Scientific Writings in Latin, Londres, 1963, col. 1185.

85. Très probablement les Problemata du pseudo-Aristote dans la traduction d'Évrart de Conty. $C f$. P. D. LeEmans et M. Goyens éd., Aristotle's Problemata in Different Times and Tongues, Louvain, 2006; et l'édition critique en cours d'élaboration par Michelle Goyens et son équipe. 
Item, 1 volume les Epistres Saint Pol, Actus apostolorum et l'Apocalipse, prisiés ensemble

xii s.

xli ${ }^{\mathrm{a}}$ grossa

Somme :

iiiii $^{\mathrm{xx}}$ xiiii 1 xvi s.

[...] [p. 41]

Lettres inventoriees le jeudi xvie dudit moys par $\mathbf{M}^{\mathrm{e}}$ Thomas Griffart et $\mathbf{M}^{\mathrm{e}}$ Jehan Hays

Premierement, une cedule en parchemin par laquelle l'evesque a present d'Arras confesse devoir audit deffunct d'or presté [?] la somme de 1 escus, donnee le vi ${ }^{\mathrm{e}}$ de fevrier iiiic $x i i$, valant

xlv 1.

Item, une cedule en parchemin, par laquelle Jehan de Lachenel, dit de Boulongne, confesse devoir audict deffunct d'or presté $\mathrm{xx}$ escus, donnee le xvii ${ }^{\mathrm{e}}$ de decembre iiiiciiii, valant

xlviii 1.

Item, $1^{\mathrm{e}}$ cedule en papier, par laquelle le cardinal de Bar confesse devoir audit deffunct la somme de c escus donnee le vi ${ }^{\mathrm{e}}$ de septembre iiiic ${ }^{\mathrm{x} v}$, valant

iiiii $^{\mathrm{xx}} \mathrm{x}$ 1. p.

Item, 1 vidimus de Chastellet, ouquel sont incorporees unes lettres par lequelles Charles de Poitiers, lors evesque de Chaalons et a present de Lengres, confesse devoir audit deffunct la somme de ii $^{c} \mathrm{xxv}$ 1. t., donnees le xxvi de fevrier iiiic vi, valant

ix ${ }^{\mathrm{xx}} 1 . \mathrm{p}$.

Item, $1^{\mathrm{e}}$ cedule en papier, par laquelle $\mathrm{M}^{\mathrm{e}}$ Jourdain Morin, chanoine de Chartres, confesse devoir audict deffunct la somme de xl escus, donnee le xxv d'octobre iiiic viii, valant

xxxvi 1 .

Item, $1^{\mathrm{e}}$ aultre cedule en papier, par laquelle ledit Morin confesse devoir audit deffunct la somme de xx escus, donnee le iiii $^{\mathrm{e}}$ de décembre iiiicix, valant

xviii 1.

Item, $1^{\mathrm{e}}$ cedule en papier de $\mathrm{M}^{\mathrm{e}}$ Jehan de Vissart, doyen de Chaalons, qui confesse devoir audit deffunct la somme de x escus, donnee le iiii ${ }^{\mathrm{e}}$ de jung iiii $^{\mathrm{c}} \mathrm{Xv}$, valant

ix 1.

Item, $1^{\mathrm{e}}$ cedule en papier, excripte de la main dudit deffunct, par laquelle il confesse avoir en gage dudit evesque de Chaalons, monseigneur Charles de Poytiers, un gobelet et $1^{\mathrm{e}}$ ayguière d'argent dorez, 1 creusequin couvert et $1^{\mathrm{e}}$ ymage de la Magdelaine pour c l. et xl s. t., par cellui deffunct prestez audit evesque. Pour ce cy: cii l.t., valant

iiii ${ }^{x x}$ 1. xii s. p.

Item, 1 brevet du Chastellet, par lequel Jehan Guynart, maire de Cretueil, confesse devoir audit deffunct xx 1. t., pour les causes contenues en ycellui, donné le $\mathrm{xi}^{\mathrm{e}}$ de mars iiii ${ }^{\mathrm{x}} \mathrm{xv}$, valant

xvi 1.p.

Item, ii aultres brevès chacun de xx 1 . t. pour ycelles causes, l'un pour $\mathrm{M}^{\mathrm{e}}$ Thomas d'Aulnoy et l'autre pour $\mathrm{M}^{\mathrm{e}}$ Guigou d'Alby, valant 
Item, $1^{\text {e }}$ lettre de Chastellet par laquelle Jehan Gaude confesse [p. 42] devoir audict deffunct la somme de $\mathrm{ii}^{\mathrm{c}}$ escus d'or de present, donnee le $\mathrm{xvi}^{\mathrm{e}}$ de novembre cccc et huit, valant $\mathrm{ix}^{\mathrm{xx}} 1 . \mathrm{p}$.

Item, 1 brevet de Chastellet par lequel Raoulet Le Roussel confesse devoir audict deffunct de present xxxvi s., donnés en date mil iiii ${ }^{\mathrm{x}} \mathrm{x}$, le lundi vii ${ }^{\mathrm{e}}$ jour de mars, signé Preudomme et Luilier, pour cecy

xxxvi s.

Item, 1 aultre brevet signé La Mote et Manessier, par lequel Jehan Osmont, demourant à Tresly au diocese de Coustances, confesse devoir audict deffunct iiii ${ }^{\mathrm{xx}}$ xii 1 . t. pour cause des arrerages de la prebende de Coustances, donné le $\mathrm{xxv}^{\mathrm{e}}$ jour de jung mil ccccxvi, pour ce cy: iiiii ${ }^{\mathrm{xx}}$ xii 1. t., valant

1xxiii 1. xii s.

Item, $1^{\mathrm{e}}$ cedule en parchemin signee Philippe Ligier, qui confesse devoir audict deffunct de present viii escus en or, donnnés le $\mathrm{xxvi}^{\mathrm{e}}$ de juing iiiic $^{\mathrm{c}} \mathrm{xii}$, pour ce cy viii escus, valant

vii 1 iiii s.

Item, $1^{\text {e }}$ cedule en papier par Marie de Roolelong et estoit signee de G. Potier qui confesse devoir audit deffunct pour les parties contenues en ycelle cedule la somme de L fr. xii s. par., pour ce cy: L fr. xii s. par., valant

xl 1. xii s.

Item, $1^{\mathrm{e}}$ cedule en papier signee de G. de Manpoyvre qui confesse devoir audict deffunct xxxix fr. de reste de L escus donné le xiii jour de juing iiiicix. Pour ce cy: xxxix 1. t. qui valent

xxxvi 1. iiii s. xliiii ${ }^{\mathrm{a}}$ grossa

Somme:

[...] [p. 45]

Aultres biens depuis trouvez et inventoriez et prisiés par ledit deffunct

Premierement, un tableau quarré, doré d'une part et paint de rouge au doz, ou quel est painte par moytié l'ymage Notre Dame tenant son enfant qui tient une cerise et l'ymage Notre Dame a une estoyle sur l'espaule, prisiee

X s.

Item, un petit tableau rond, doré d'une part et paint de vermeil encré d'autre part, ouquel est painte Notre Dame tenant un livre et son enfant qui se baigne, et Joseph soufle le feu, prisié

iiii s.

Item, ii hanaps et ii salières d'alebastre, prisiees ensemble

viii s.

Item, $1^{\mathrm{e}}$ clochete de metal, prisiee

xii s.

Item, $1^{\mathrm{e}}$ nate de jonc marin pareille a celle de la sale cy dessus inventoriee, prisiee

xii s. [p. 46]

Item, 1 tableau de boys, ouquel est figuree la teste de Philippe, duc de Bourgogne, prisiee

iiii s. 
Item, une mappemonde en parchemin mise en 1 fourrel de cuir, lequel est armoryé aux armes du duc de Bourgogne avec un compas de layton doré, non prisiez pour ce que on tient que tout est au duc de Bourgogne.

Item, une aultre mappemonde en parchemin de petit volume, non prisiee pour ce qu'elle est connue de nulle valeur.

Item, un petit coffret quarré paint, ouquel sont dedens les xii signes figurez et les mouvemens du soleil et de la lune, non prisiee comme dessus.

Item, trois astralabes de layton, dequels l'un est brisié, non prisiés.

Item, un petit poulpite de pié et demi de long, ouquel il a une ardoise enchacee, prisié

iiii s.

Item, 1 tableau rond ouquel est la calculacion a doubler les poins de l'escequier per grains de fourment, prisé

Item, 1 tableau rond ou les xii signes sont escrips d'encre et de vermeille sur parchemin, non prisié par sa petite valeur.

Item, 1 vieil astralabe de boys en 1 estuy de cuir, non prisié pour la cause dessusdite.

Item, iii chapelles de plomb a faces caves, la greigneur xii s., la moyenne x s. et la petite vi s., valant

xxviii s.

Item, $1^{\mathrm{e}}$ grant huye enchacillee et ii tresteaux, l'uye prisee $\mathrm{x}$ s. et les tresteaux iiii s., valant

xiiii $\mathrm{s}$

Item, furent trouvez en un coffre de la chambre vert cii blaus de viii d. la piece

xii 1 .

xlviii ${ }^{\mathrm{a}}$ grossa

Somme :

xvi 1. xviii s. p.

\section{Document 4}

\section{La disposition de l'hôtel de Jean Durand selon le déroulement de l'inventaire après décès}

Du samedi 4 juillet au [mercredi] 8 juillet

[L'inventaire commence par le relevé du contenu de divers coffres où se trouvaient des sommes d'argent et des objets précieux: bijoux, liste de la vaisselle dorée et d'argent. Ces objets étaient répartis en plusieurs pièces de l'hôtel, qui ne sont pas indiquées («oudit hostel en plusieurs places »)].

[Jeudi] 9 juillet [commence la présentation du contenu des différentes pièces de l'hôtel]

En la salle basse

(meubles, eschiquier)

En la galerie du jardin

(meubles)

Ou jardin dudit hostel

(bèche, échelle, «refredouer») 
En la basse chambre pavee sur le jardin

Ou parlouer de l'ostel devant la cour

En la cuisine En la haulte galerie prés la grant haulte salle au
bout des grans degrez

En la haulte sale dudit hostel

En la petite chambre emprés d'ycelle sur le (banc,tissus) jardin

En la chambre vermeille

En la petite estude sus la terrasse

Ou retrait en hault emprés la chapelle

En la dicte chapelle

En la cave

Ou celier dudist hostel sur la cave

En la haulte chambre pavee

En une haulte chambre lambroyssiee de plastre

Ou grenier au foin

En l'allee devant le grenier

En la place joignant ladicte allee devant la (meubles, tissus, nappes) chambre blanche

En la chambre blanche (meubles, vaisselle)

(meubles)

(casseroles, outils, meubles)

(meuble, 1 banc)

(banc, fauteuil...)

(banc, meubles...)

(meubles, banc, fauteuil)

(banc, tapis)

(tableau, nappes et autres parements liturgiques, chandeliers)

(vins)

(vins)

(meubles, bois)

(coussin, écrin)

(1 table)

(tissus, chassis)

(couvre-lits, tissus, escabeau, meubles, matelas, 1 coffre contenant des draps)

Samedi 11 juillet

En la garderobe joignant ladite chambre (tissus de prix, tapisserie, tapis, blanche meubles)

Le mesme jour aprés dîner, toujours dans la (vêtements, fourrures, un harnais garde robe à mulet, un coffre avec des tissus de valeur)

En le scriptouere emprés ladicte chambre (armoire, coffre, gants, 1 blanche ceinture)

Ou retrait de ledit scriptouere (coffre, 1 chaire à dossier)

En l'estude dudit hostel (un coffre contenant de nappes ouvragées, un comptoir, un autel portatif, un tapis de haute lice)

Dimanche [12] et lundi [13 juillet]: pas d'inventaire 
Mardi 14 juillet

En la chambre vert

(table, écran, fauteuil, couverture tapissé, carreaux décorés, buffet)

En la garderobe prés de la dite chambre vert (oreillers, chaperons, houppelandes, manteaux)

En la grande chambre sur l'estable (banc, dressoir, fauteuil, couvertures, tapis, rideaux)

En la garderobe de ladite chambre (coffre, tréteaux, chaise, lit)

Mardi 14 après dîner

Oudit perlouer dudit hostel

(chandeliers)

En l'estable

(selle, frein à mule, couverture)

En la chambre des vailés sur la court

(lits, coussin)

En la chambre sus la chambre aux vailès

(table, lit, coussin, couvertures...)

Ensuivent les livres trouvez audit hostel

Le linge venant de la buee

(nappes)

Autres bien depuis trouvez

(vêtements, bijoux, avoine)

Jeudi 16 juillet

Lettres inventoriees

Autres biens trouvez oudit hostel

(chandeliers, couteaux, vêtements, tissus)

Aultres biens trouvez et venus a connaissance puis ledit inventoire fait

(vin, maison à Vannes, une lèchefrite, couverture, drap à l'hostel)

[Ici est mentionnée la vente de l'hôtel à Nicolas de Baye.]

Autres sommes de deniers dues à cette exécution (mention de prébendes, documents)

Autres biens depuis trouvez et inventoriez et (tableaux, clochette de métal, prisiés par ledit deffunct tableau figurant le duc de Bourgogne, «mappemondes», instruments d'astronomie) 


\section{Langues d'Angleterre,}

coordonné par Alban Gautier et Jean-Pascal Pouzet

5 Alban Gautier et Jean-Pascal Pouzet

Les langues de

l'Angleterre médiévale : au-delà du bilinguisme

\section{David Trotter}

Peut-on parler de judéoanglo-normand?

Textes anglo-normands en écriture hébraíque

35 Christopher Lucken

Le beau français

d'Angleterre. Altérité de l'anglo-normand et invention du bon usage

57 Aude Mairey John Gower ou le multilinguisme en action

73 Catherine Nall et Daniel Wakelin Le déclin du multilinguisme dans The Boke of Noblesse et son Codicille de William Worcester
Essais et recherches

93 Donatella Nebbiai

Les livres de Jean

Durand († 1416),

"physicien »

et astrologue

\section{Lucie Laumonier}

En prévision des vieux

jours: les personnes âgées à Montpellier à la fin du Moyen Âge

\section{Points de vue}

147 François Foronda Procès politiques: une manie française?

161 Clément Lenoble Monnaie, valeur et citoyenneté chez Olivi et Eiximenis. "Moralisation de l'économie » ou "économie politique" médiévale?

181 Notes de lecture 205 Livres reçus 